\title{
Radiation from shock-accelerated particles
}

\author{
K.-I. Nishikawa ${ }^{* a^{\dagger}}$, E. J. Choi ${ }^{b}$, K. W. Min ${ }^{b}$, J. Niemiec ${ }^{c}$, B. Zhang ${ }^{d}$, P. Hardee ${ }^{e}$, Y. \\ Mizuno $^{f}$, M. Medvedev ${ }^{g}$, A. Nordlund ${ }^{h}$, J. Frederiksen ${ }^{h}$, H. Sol ${ }^{i}$, M. Pohl ${ }^{j}$, D. H. \\ Hartmann $^{k}$, and G.J. Fishman ${ }^{l}$ \\ ${ }^{*}$ Center for Space Plasma and Aeronomic Research, University of Alabama in Huntsville, \\ Huntsville, AL 35805, USA \\ ${ }^{b}$ Korea Advanced Institute of Science and Technology, Daejeon 305-701, South Korea \\ ${ }^{c}$ Institute of Nuclear Physics PAN, ul. Radzikowskiego 152, 31-342 Krakow, Poland \\ ${ }^{d}$ Department of Physics and Astronomy, University of Nevada, Las Vegas, NV 89154, USA \\ ${ }^{e}$ Department of Physics \& Astronomy, The University of Alabama, Tuscaloosa, AL 35487, USA \\ ${ }^{f}$ Institute of Astronomy, National Tsing-Hua University, Hsinchu, Taiwan 30013, R.O.C. \\ ${ }^{g}$ Department of Physics and Astronomy, University of Kansas, KS 66045, USA \\ ${ }^{h}$ Niels Bohr Institute, Juliane Maries Vej 30, 2100 K£benhavn ŕ, Denmark \\ ${ }^{i}$ LUTH, Observatore de Paris-Meudon, 5 place Jules Jansen, 92195 Meudon Cedex, France \\ ${ }^{j}$ Institut für Physik und Astronomie, Universität Potsdam, 14476 Potsdam-Golm, Germany \\ ${ }^{k}$ Department of Physics and Astronomy, Clemson University, Clemson, SC 29634, USA \\ ${ }^{l}$ NASA/MSFC, 320 Sparkman Drive, Huntsville, AL 35805, USA
}

Plasma instabilities excited in collisionless shocks are responsible for particle acceleration, generation of magnetic fields, and associated radiation. We have investigated the particle acceleration and shock structure associated with an unmagnetized relativistic jet propagating into an unmagnetized plasma. Cold jet electrons are thermalized and slowed while the ambient electrons are swept up to create a partially developed hydrodynamic-like shock structure. The shock structure depends on the composition of the jet and ambient plasma (electron-positron or electron-ions). Strong electromagnetic fields are generated in the reverse, jet shock and provide an emission site. These magnetic fields contribute to the electron's transverse deflection behind the shock. We have calculated, self-consistently, the radiation from electrons accelerated in the turbulent magnetic fields. We found that the synthetic spectra depend on the Lorentz factor of the jet, its thermal temperature and strength of the generated magnetic fields. The detailed properties of the radiation are important for understanding the complex time evolution and/or spectral structure in gamma-ray bursts, relativistic jet shocks, and supernova remnants.

Gamma-Ray Bursts 2012 Conference-GRB2012,

May 07-11, 2012

Munich, Germany

\footnotetext{
${ }^{*}$ Speaker.

†E-mail: ken-ichi.nishikawa@nasa.gov
} 


\section{Introduction and Simulations}

Particle-in-cell (PIC) simulations can reveal the physical mechanism of particle acceleration that occurs in the complicated dynamics within relativistic shocks. Recent PIC simulations of relativistic electron-ion and electron-positron jets injected into an ambient plasma show that acceleration occurs within the downstream jet [4].

The effects of different mass ratios $\left(m_{\mathrm{i}} / m_{\mathrm{e}}=1,20\right)$ are shown in Figure 1[2]. Figure 1(b, and d) are from an electron-positron simulation as compared to an ion-electron simulation shown in Figure 1(a, and c). Forward and reverse shock structure is generated in both cases but differences in the shape of the generated shock structure and in the magnetic fields are immediately apparent. In the electron-ion case a sharp contact discontinuity (CD) with strongly enhanced magnetic field is produced (Figs. 1a and 1c). In the electron-positron case the maximum associated with the forward shock is significantly increased. In this case, both positrons and electrons in the ambient appear swept up by the front of the jet without the strong interactions seen in the electron-ion case, and the jet density exhibits a small peak associated with the shocked ambient particles. For both electronion and pair plasmas cases magnetic fields shown in Fig 1 (c, and d) increase in the reverse shock region, but the maximum magnetic field occurs at the contact discontinuity (CD) in the electron-ion case. Magnetic (Figs. 1c and 1d) and electric field (not shown) in the transverse directions strongly fluctuate in this region.
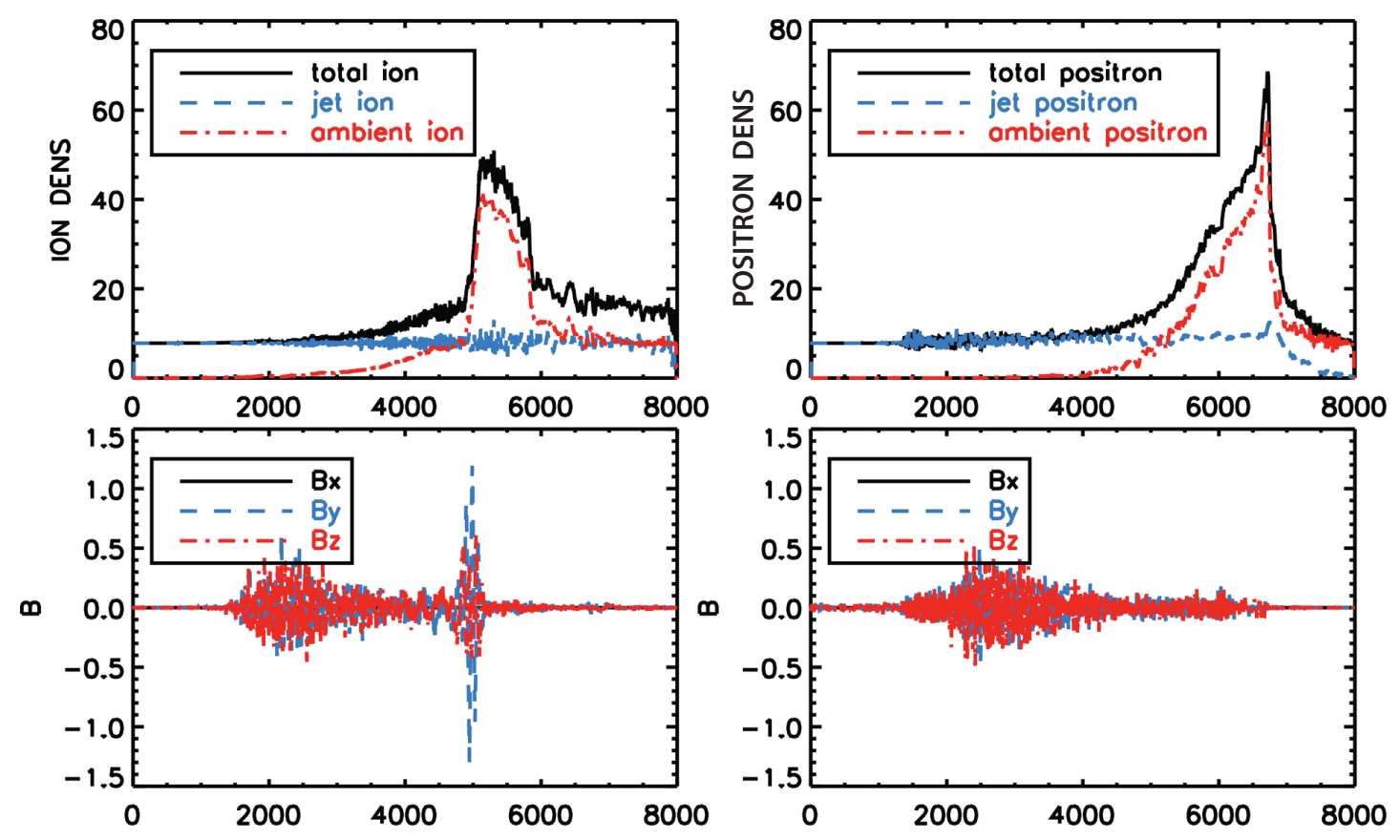

Figure 1: Snap shots of ion density (a), positron density (b), and magnetic field strength, $B$, ((c) for electronion plasma, and (d) for pair plasma) at $\omega_{\mathrm{pi}} t=375$. 


\section{Synthetic spectra from simulations}

We have calculated the radiation spectra directly from our simulations by integrating the expression for the retarded power, derived from the Liénard-Wiechert potentials for a large number of representative particles in the PIC representation of the plasma[5]). In order to obtain the spectrum of the synchrotron/jitter emission, we consider an ensemble of electrons selected in the region where the Weibel instability has fully grown and where the electrons are accelerated in the selfconsistently generated magnetic fields.

Figure 2 shows how our synthetic spectrum matches with spectra obtained from Fermi observations. Figure 2a shows the observed spectra in $v F_{v}$ as modeled by[1] at five different times. The
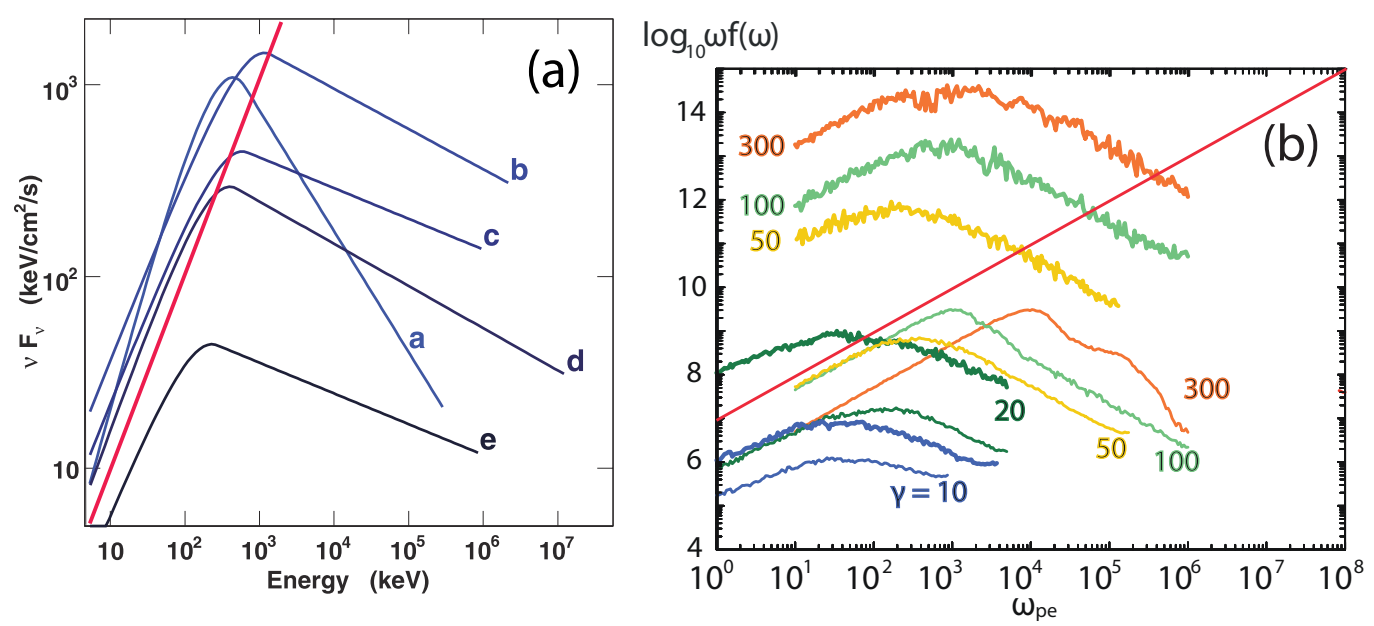

Figure 2: Comparison of synthetic spectra (b) with Fermi observations for GRB 080916C (a). Figure 2a shows modeled Fermi spectra in $v F_{v}$ units at five different times. A flat spectrum would indicate equal energy per decade in photon energy. The changing shapes show the evolution of the spectrum over time. Figure $2 \mathrm{~b}$ shows PIC generated spectra for jet Lorentz factors of $\gamma=10,20,50,100$, and 300. For each case we show two simulations represented by the same color for cold (thin, lower lines) and warm (thick, upper lines) jet electrons. The low frequency slope is approximately 1.

red lines in Fig. 2a and 2b indicate a slope of one, and except for the Fermi spectrum at time "a" the low frequency slopes are all approximately one. As shown in Fig. 2a, prompt emission spectra of GRB 080916C exhibit a remarkably constant shape (low-energy and hi-energy slopes are different but do not evolve significantly). On the other hand the peaks of the SED vary significantly with time. Based on our PIC simulations, this behavior can be interpreted in terms of declining bulk Lorentz factor.

Behind the reverse shock electrons are accelerated and strong magnetic fields are generated as shown in Fig. [4, 2]. Therefore, this region would seem more likely to produce the emission that is observed. In the future we will examine PIC computed spectral changes over time varying plasma conditions such as plasma composition and ambient magnetic fields along with jet Lorentz factors and jet thermal temperatures. We are currently simulating much larger systems to obtain synthetic spectra at different times in the nonlinear development where different particle acceleration rates and magnetic field strengths apply. 


\section{Discussion}

A double shock structure (bow and jet shocks separated by a contact discontinuity region) is formed and electrons can be accelerated due to the Weibel instability in both shocks. Since we calculate the radiation from the electrons in the observer frame calculated spectra can be compared directly with observations. As shown in Fig. 1, the strongest electron acceleration and strongest magnetic fields are generated in the reverse, jet shock. Therefore, in this simulation this region would produce the emission that is observed.

Emission obtained using the method described above is obtained self-consistently, and automatically accounts for magnetic field structures on the small scales responsible for jitter emission. By performing such calculations for simulations using different parameters, we can investigate and compare the different regimes of jitter- and synchrotron-type emission[3]. This addresses the observed low frequency GRB spectral index violation of the synchrotron spectrum line of death[3].

More extended simulations are required for detailed comparisons with GRB spectra. In particular, the nonlinear development of the Weibel instability must be followed for a sufficiently long time, and radiation spectra must be sampled for the various plasma conditions suggested by dynamic models of jet evolution.

\section{Achknowledgments}

This work is supported by NSF-AST-0506719, AST-0506666, AST-0908040, AST-0908010, NASANNG05GK73G, NNX07AJ88G, NNX08AG83G, NNX08AL39G, and NNX09AD 16G. JN was supported by MNiSW research project N N203 393034, and The Foundation for Polish Science through the HOMING program, which is supported through the EEA Financial Mechanism. Simulations were performed at the Columbia facility at NASA Advanced Supercomputing (NAS), and on the IBM p690 (Copper) at the National Center for Supercomputing Applications (NCSA) which is supported by the NSF. Part of this work was done while K.-I. N. was visiting the Niels Bohr Institute. Support from the Danish Natural Science Research Council is gratefully acknowledged. This report was finalized during the program "Particle Acceleration in Astrophysical Plasmas" at the Kavli Institute for Theoretical Physics which is supported by the National Science Foundation under Grant No. PHY05-51164.

\section{References}

[1] A.A. Abdo, et al. Fermi Observations of High-Energy Gamma-Ray Emission from GRB 080916C, Science 323 (2009) 1688.

[2] E. J. Choi, K. Min, K.-I. Nishkawa, and C. R. Choi, Magnetic field effects on shock evolution and particle acceleration, ApJ in preparation (2012).

[3] M. V. Medvedev, The theory of spectral evolution of the GRB prompt emission, ApJ 637 (2006) 869.

[4] K.-I. Nishikawa, J. Niemiec, P. E. Hardee, M. Medvedev, H. Sol, Y. Mizuno, B. Zhang, M. Pohl, M. Oka, \& D. H. Hartmann, Weibel instability and associated strong fields in a fully $3 D$ simulation of a relativistic shock, ApJ 689 (2009) L10.

[5] K.-I. Nishikawa, J. Niemiec, M. Medvedev, B. Zhang, P. E. Hardee, Å. Nordlund, J. Frederiksen, Y. Mizuno, H. Sol, M. Pohl, D. H. Hartmann, \& G. J. Fishman,

Radiation from relativistic shocks with turbulent magnetic fields, AdvSR 47 (2011) 1434. 